\title{
On the age and mass function of the globular cluster M 4: A different interpretation of recent deep HST observations ${ }^{\star}$
}

\author{
G. De Marchi ${ }^{1}$, F. Paresce ${ }^{2}$, O. Straniero ${ }^{3}$, and P. G. Prada Moroni ${ }^{4,5,6}$ \\ ${ }^{1}$ European Space Agency, Space Telescope Operations Division, 3700 San Martin Drive, Baltimore MD 21218, USA \\ ${ }^{2}$ European Southern Observatory, Karl-Schwarzschild-Str. 2, 85748 Garching, Germany \\ e-mail: fparesce@eso.org \\ 3 Osservatorio Astronomico di Teramo, Via Maggini, 64100 Teramo, Italy \\ e-mail: straniero@te.astro.it \\ ${ }^{4}$ Dipartimento di Fisica, Università di Pisa, Via Buonarroti 2, 56127 Pisa, Italy \\ e-mail: prada@df.unipi.it \\ 5 Istituto Nazionale di Fisica Nucleare, Sezione di Pisa, 56010 Pisa, Italy \\ ${ }^{6}$ Osservatorio Astronomico di Teramo, Via Maggini, 64100 Teramo, Italy
}

Received 2 June 2003 / Accepted 30 September 2003

\begin{abstract}
Very deep images of the Galactic globular cluster M4 (NGC6121) through the F606W and F814W filters were taken in 2001 with the WFPC2 on board the HST. A first published analysis of this data set (Richer et al. 2002) produced the result that the age of M4 is $12.7 \pm 0.7 \mathrm{Gyr}$ (Hansen et al. 2002), thus setting a robust lower limit to the age of the universe. In view of the great astronomical importance of getting this number right, we have subjected the same data set to the simplest possible photometric analysis that completely avoids uncertain assumptions about the origin of the detected sources. This analysis clearly reveals both a thin main sequence, from which can be deduced the deepest statistically complete mass function yet determined for a globular cluster, and a white dwarf (WD) sequence extending all the way down to the $5 \sigma$ detection limit at $I \simeq 27$. The WD sequence is abruptly terminated at exactly this limit as expected by detection statistics. Using our most recent theoretical WD models (Prada Moroni \& Straniero 2002) to obtain the expected WD sequence for different ages in the observed bandpasses, we find that the data so far obtained do not reach the peak of the WD luminosity function, thus only allowing one to set a lower limit to the age of M 4 of $\sim 9$ Gyr. Thus, the problem of determining the absolute age of a globular cluster and, therefore, the onset of GC formation with cosmologically significant accuracy remains completely open. Only observations several magnitudes deeper than the limit obtained so far would allow one to approach this objective.
\end{abstract}

Key words. globular clusters: individual: M4 - stars: white dwarfs - stars: low-mass, brown dwarfs stars: luminosity function, mass function - cosmology: cosmological parameters

\section{Introduction}

Stars in the metal-poor globular clusters (GCs) of our Galaxy's halo are currently the oldest objects known whose age can be determined by present means, thereby setting a firm lower limit on the age of the Universe and allowing independent confirmation by other means of the age recently obtained with WMAP (Bennett et al. 2003). The best measurements so far based on main sequence (MS) fitting yield a value of the age of the oldest GCs of $12.5 \mathrm{Gyr}$ with a $95 \%$ confidence range of $2.5 \mathrm{Gyr}$ (Krauss 2001; Gratton et al. 2003), with the largest contribution to the measurement error coming from the distance uncertainty.

Send offprint requests to: G. De Marchi,

e-mail: gdemarchi@rssd.esa.int

* Based on observations with the NASA/ESA Hubble Space Telescope, obtained at the Space Telescope Science Institute, which is operated by AURA for NASA under contract NAS5-26555.
Taken at face value, this number compares favourably with the expansion age of the Universe implied by WMAP (Bennett et al. 2003) and by the most recent $H_{0}$ and geometry measurements (13 \pm 3 Gyr; Lahav 2001) as well as with the results of radioactive dating of a very metal-poor star $(12.5 \pm 3.0 \mathrm{Gyr}$; Cayrel et al. 2001).

A comparison between the measured expansion age of the universe and the GC limit will also shed light on the presently uncertain and controversial issue of the time of formation of the oldest halo GCs (Gnedin et al. 2001). Current estimates of the epoch of formation of these objects range from a minimum of 10-100 Myr, if they were formed at the epoch of recombination, to a maximum of $5 \mathrm{Gyr}$, if they were formed as the result of thermal instabilities in the Galactic halo (Fall \& Rees 1985). This enormous range can be significantly reduced with a precise measurement of a GC age which, in turn, might help us to determine, for example, whether the Galactic halo formed from 
the accretion of dwarf galaxies or from proto-galactic cloud collapse (Mould 1998). What is really needed is a GC age measurement in the range $10-15 \mathrm{Gyr}$ with a $2 \sigma$ uncertainty of $10 \%$ or less, comparable to the current precision on $H_{0}$.

Since the main obstacle by far to a more precise determination of GC ages lies in the uncertainty on their distance, the situation is unlikely to change significantly until well after the launch of GAIA and SIM in the next decade. In this regard, WDs could play an important rôle both as distance indicators and as cosmo-chronometers and allow measurements more accurate than the MS turn-off method. As recognised early on by Mestel (1952), the decrease of the WD brightness with time is the result of a cooling process so that the luminosity of a WD indicates its age. Unlike the turn off age-luminosity relationship, the cooling timescale is independent of the original chemical composition of the progenitor star. Cooling is generally rather fast, except during the crystallisation of the core that lasts for several Gyr. Thus, a pile up of WDs is expected in the CMD of an old stellar system. In practice, the WD luminosity function (LF) should present a peak corresponding to the portion of WDs close to the end of their crystallisation phase, followed by a sharp cutoff. The luminosity of this peak is a powerful age indicator that has been already used to date the Galactic disc (Leggett et al. 1998) as well as some open clusters (Von Hippel \& Gilmore 2000; Richer et al. 1998). Owing to the intrinsic faintness at which the WD LF peaks in very old stellar systems, the extension of this method to most GCs has remained thus far just a dream (see Fontaine et al. 2001 for a detailed discussion of the present state of the art). At least for the nearest GCs, however, this goal is within the reach of the HST. Indeed, using deep observations of the WD cooling sequence of M 4 obtained with the WFPC2 camera, Hansen et al. (2002) have reported a determination of the age of the cluster M 4 to be $12.7 \mathrm{Gyr}$ to within $\pm 0.7 \mathrm{Gyr}$ at the $2 \sigma$ level, thus attaining that accuracy needed to set meaningful constraints on the age of the universe, as well as concordance with it.

Obviously, reliable ages can be obtained only if an adequate calibration of the age-luminosity relationship is available. This problem has been recently reviewed by Prada Moroni \& Straniero (2002; hereafter PMS02). They showed that, although in principle cosmo-chronology based on WDs is a promising tool, in practice the large discrepancies amongst the recently published theoretical cooling sequences imply that a firm calibration of the age-luminosity relationship is not yet available, especially for the range of ages suitable for GCs. The main reason for the quoted discrepancies is the large uncertainty in the input physics needed to model the WD structure and its evolution. In particular, models depart progressively from one another at low luminosities (see Fig. 1 in PMS02), where they are very sensitive to the details of the physics of WD interiors and, thus, provide ages that can vary by as much as $3 \mathrm{Gyr}$ at $\log L / L_{\odot}=-5.5$. The key issue is that, since the cooling ages predicted by different models begin to depart considerably from one another for luminosities $\log L / L_{\odot} \lesssim-4.5$, any hope to discriminate between competing theories by verifying the validity of their predictions rests on our ability of securing a statistically complete sample of WDs fainter than that luminosity.

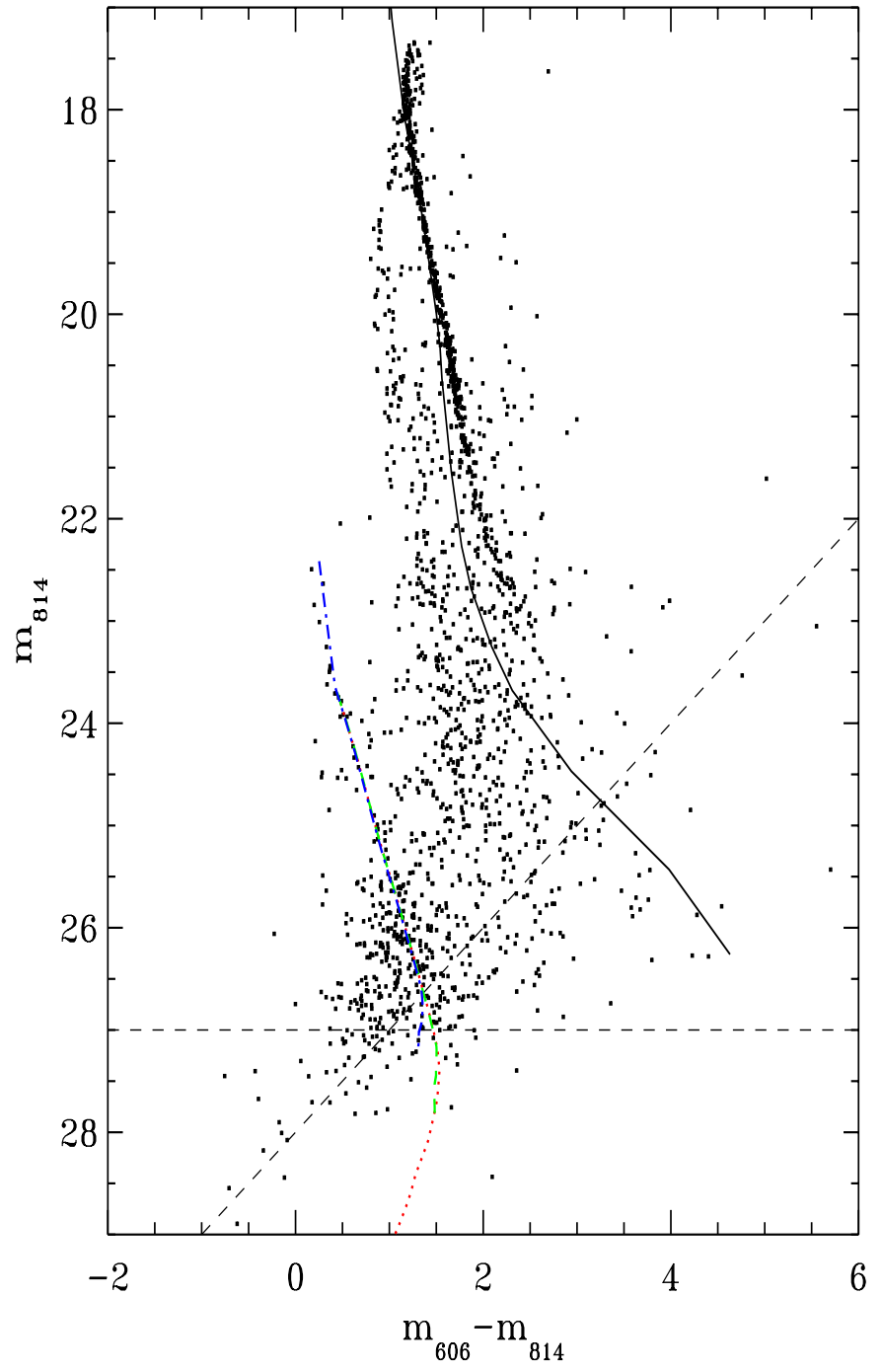

Fig. 1. Colour-magnitude diagram of the three WF chips. The dashed lines indicate the $5 \sigma$ detection level, corresponding in practice to $50 \%$ completeness. The solid line next to the cluster MS corresponds to the isochrone for an age of $10 \mathrm{Gyr}$ and metal content $[\mathrm{M} / \mathrm{H}]=-1$ (Baraffe et al. 1997). The coloured lines drawn through the WD region correspond to isochrones for ages of $8 \mathrm{Gyr}$ (blue, dot-dashed), $10 \mathrm{Gyr}$ (green, dashed) and 12 Gyr (red, dotted).

We have, therefore, subjected the same data used by Hansen et al. (2002) to an independent scrutiny to verify at which level of significance they allow one to accept or reject a different set of WD cooling models, namely those of PMS02.

\section{The data}

The data used in this paper have been obtained with the WFPC2 on board the HST as part of programme 8679 and are briefly described in Richer et al. (2002). The target is a region located $\sim 5^{\prime} \mathrm{E}$ of the nominal centre of M 4 and has been imaged through the F606W filter (98 images of duration $1300 \mathrm{~s}$ each) and in the F814W filter (148 images each of duration $1300 \mathrm{~s}$ ). Displacements of a fraction of a pixel (dithering) have been applied between subsequent exposures in order to improve the sampling of the point spread function (PSF) and to mitigate the 
effects of cosmetic defects (Hook \& Fruchter 2000). We have retrieved the complete dataset from the ESO/ST-ECF archive by making use of the recalibration on-the-fly and automated association options (Micol \& Durand 2002) to register and combine all images in the same bandpass in a fully automated way. The accuracy of the registration and combination procedures has been verified by comparing the properties of the PSF in the individual images and in the combined ones. We find that the full width at half maximum (FWHM) of stars in the frame increases by about $15 \%$, as is typical of any shift-and-add operation conducted on under-sampled images. A known feature of this automated processing is the incorrect subtraction of a constant sky from the final image, which has, however, no effect on the accuracy of our measurements, since the latter have been carried out via aperture photometry with the background estimated locally in an annulus surrounding each object.

The equivalent exposure time of the final combined images (127400 s in F606W and $192400 \mathrm{~s}$ in F814W) is such that stars with colour $m_{606}-m_{814} \simeq 1$ have similar signal-to-noise ratio (SNR) in both bands. To make the detection of both blue and red stars more robust, we have registered the final images with one another and run the IRAF apphot.daofind routine on the averaged frame. It is our practice to conservatively set the detection threshold at $5 \sigma$ above the local average background level to ensure that most noise spikes and PSF artefacts are automatically rejected. In this case, however, since we had combined the final F606W and F814W frames, we decided to lower the limit to $4 \sigma$ so as to avoid that very blue or very red stars would be missed in the average image which could have otherwise been detected at the $5 \sigma$ level in at least one of the two individual frames. We then carefully examined by eye each individual object detected by daofind and discarded saturated stars, a number of features even above $4 \sigma$ (PSF tendrils, noise spikes, etc.) that daofind had interpreted as stars, as well as several extended objects (with FWHM larger than twice that typical of point sources). The number of well defined objects detected in this way amounts to $\sim 1730$ in the three WF chips (we did not reduce the PC frame for it contains very few stars and would add minimally to the statistics).

Crowding not being severe, stellar fluxes were measured by using the standard digiphot.apphot IRAF aperture photometry routine, following the prescription of the "core aperture photometry" technique described in De Marchi et al. (1993). In particular, we adopted an aperture radius of 2 pixel and a background annulus extending from 3 to 5 pixel in radius. Aperture corrections were calculated for an infinite aperture and the instrumental magnitudes calibrated in the HST magnitude system (VEGAMAG) by adopting the zero points listed in the January 2002 edition of the HST Data Handbook (Mobasher et al. 2002). The photometric uncertainty ranges from $\lessgtr 0.01 \mathrm{mag}$ at $m_{606} \simeq 19, m_{814} \simeq 18$ to $\sim 0.35 \mathrm{mag}$ at $m_{606} \simeq 28.5$ and $\sim 0.25$ mag at $m_{814} \simeq 27$. The photometric uncertainties in each band, combined in quadrature, provide the error on the colour.

To assess in a statistical way the completeness of our photometry, we have run artificial star tests by adding, in repeated trials, several fake stars of known brightness and subjecting each artificial image to the same star finding and aperture photometry routines used for the science frames. Because of the conservative detection threshold adopted ( $4 \sigma$ above the local background), an artificial star is always recovered unless it overlaps with a brighter feature, such as another star, an extended object, a spider ghost, a noise spike or other feature. The completeness is, therefore, set at any magnitude by the fraction of area which is free of brighter objects. The recovery fraction is always larger than $\sim 95 \%$ above $m_{814}=24.5$ and slowly decreases to $\sim 80 \%$ at $m_{814}=25.7$ and to $\sim 50 \%$ at $m_{814}=26.8$. The difference between the input and recovery magnitudes is consistent with the photometric errors quoted above.

\section{The main sequence colour-magnitude diagram and luminosity function}

From the photometry obtained as explained in the previous section, we derive the colour-magnitude diagram (CMD) shown in Fig. 1. The dashed lines mark, for both bands, the magnitude limit at which the photometry reaches the $50 \%$ completeness level and happen to correspond, in this particular case, to those objects whose central PSF pixel rises above the local background level by 5 times the standard deviation of the latter. This condition is, therefore, more stringent than that set by the $4 \sigma$ detection limit and suggests that we exclude from the analysis that follows the objects below the dashed lines, since their statistical significance would be anyhow too uncertain. The cluster MS is narrow and well defined in the range $17.5 \lessgtr m_{814} \lesssim 23$. At the bright end, the MS is truncated because of saturation (which, at least for the central PSF pixel, extends up to $m_{814} \simeq 19.3$ ), whilst below $m_{814} \simeq 23.5$ it becomes practically indistinguishable from field stars (which, at that magnitude, span rather uniformly the colour range $1 \lesssim$ $m_{606}-m_{814} \lesssim 3$ to the left of the MS), well before the onset of any significant appreciable photometric incompleteness.

The solid line drawn next to the MS represents the theoretical isochrone for an age of $10 \mathrm{Gyr}$ and metallicity of $[\mathrm{M} / \mathrm{H}]=$ -1 as obtained by Baraffe et al. (1997) in the specific bandpasses used here for a distance modulus $(m-M)_{V}=12.83$ and colour excess $E(B-V)=0.36$ (Harris 1996), which in turn imply $(m-M)_{I}=12.25$. As already noticed by Bedin et al. (2001), who analysed shallower exposures of the same area of M 4 collected with the WFPC2 in the F555W and F814W filters, the match between the observed and predicted MS is rather poor. They attribute this discrepancy to the inadequate treatment of the atmospheric opacity in the $V$ band (F555W) compared to redder colours, yet we find that the situation does not improve even when the redder F606W band is used instead of the F555W one. By shortening the distance modulus of about $\sim 0.5 \mathrm{mag}$, one could better fit the lower portion of the MS, but the isochrone would then fail to reproduce the observations at the bright end, where models are known to be accurate. Adopting a different set of theoretical models (Cassisi et al. 2000; Montalban et al. 2000) does not improve the fit. Certainly, a trustworthy isochrone drawn in the plane of the observed colours would facilitate the selection of true MS stars even at faint magnitudes, below $m_{814} \simeq 23$, where the MS ridge line becomes increasingly uncertain. Sophisticated techniques exist which use proper motion information from data collected 

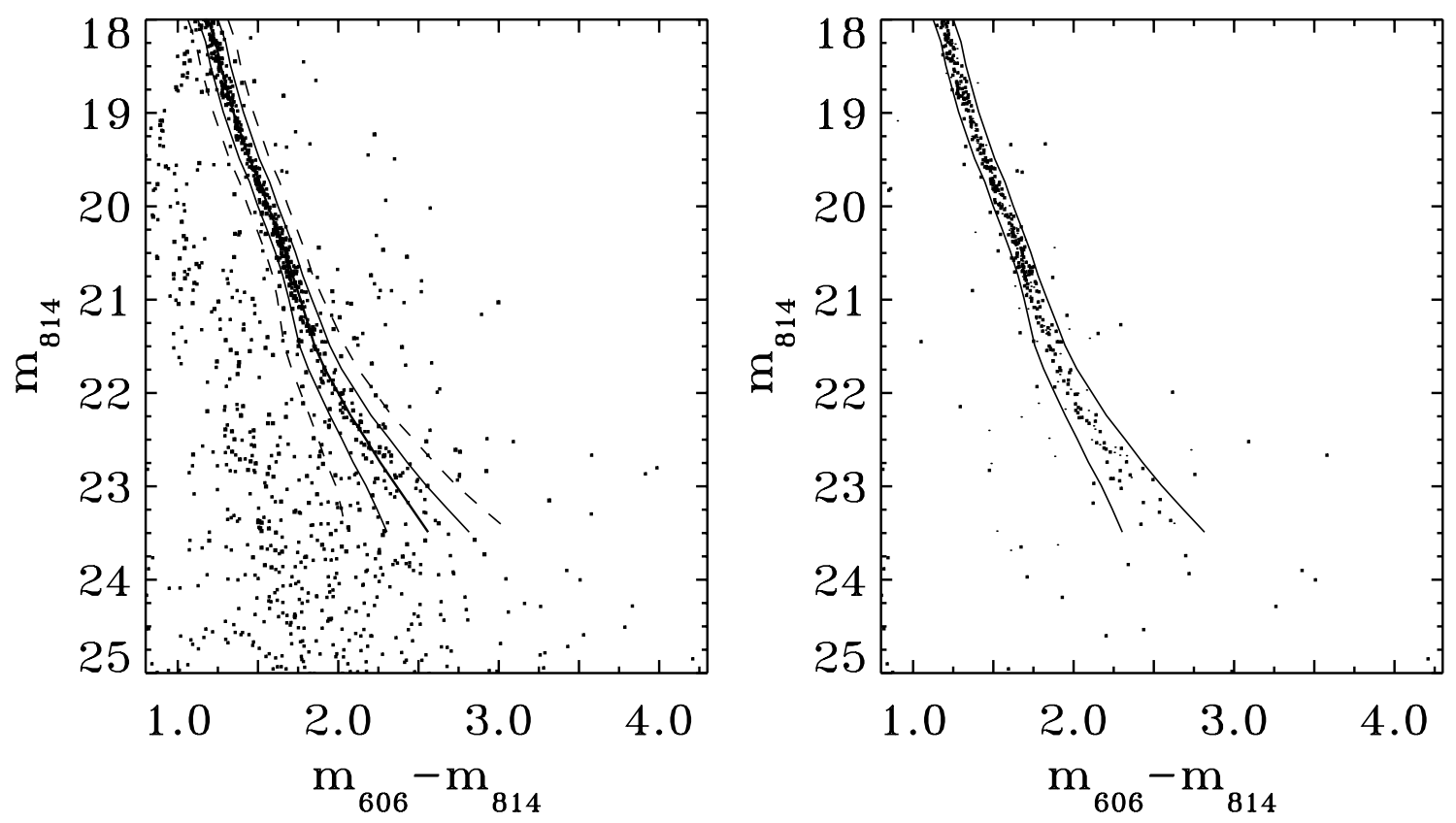

Fig. 2. a) (left panel): the thick solid line cutting through the MS represents the ridge line obtained as explained in the text. To measure the LF, we have counted the number of stars inside the thin solid lines surrounding the MS ( $\pm 4 \sigma$ band) and subtracted those found in the adjacent bands enclosed by the dashed lines (also $4 \sigma$ wide each). b) (right panel): same as (a) but showing only the stars with proper motion less than 0 '.03 (i.e. 0.3 WF pixel) between the two epochs.

at different epochs to isolate bona-fide cluster objects from field stars (see e.g. King et al. 1998; Richer et al. 2002 for the same dataset discussed here). As we show in the following, however, even with this technique the sparseness of the MS and the lack of knowledge of its colour hamper the characterisation of its lower end.

We use the colour and magnitude information contained in the CMD shown in Fig. 1 to measure the LF for MS stars. We first determine the MS ridge line by searching for the mode of the colour distribution as a function of the magnitude. We use magnitude bins whose size increases progressively from $0.2 \mathrm{mag}$ at $m_{814}=19$ to $0.4 \mathrm{mag}$ at $m_{814}=23.5$ and a step along the magnitude axis equal to half the size of the bin. The size of the bins has been selected in this way so that the number of stars sampled is approximately constant, thus minimising statistical fluctuations. An eye-ball fit through the ridge line points found in this way is shown in Fig. 2a (thick solid line). To count the number of stars along the MS we define a band, around the ridge line, whose width is $\pm 4 \sigma$ (thin solid lines). For $m_{814}>21$, we take $\sigma$ to be the uncertainty on the $m_{606}-m_{814}$ colour, whereas at brighter magnitudes we take $\sigma=0.013$, namely the colour uncertainty at $m_{814}=21$. To account for field star contamination, the number of objects found inside this band is reduced by the number of those detected within the surrounding bands (dashed lines in Fig. 2a), each $4 \sigma$ in width.

The LF obtained in this way is plotted in Fig. 3 (squares) as a function of the absolute (lower axis) and apparent (upper axis) $m_{814}$ magnitude. No correction for incompleteness is applied, since the latter is negligible, as stated in Sect. 2. Error bars reflect the Poisson statistics of the counting process, both on the MS and contaminating stars. As previously noted, at

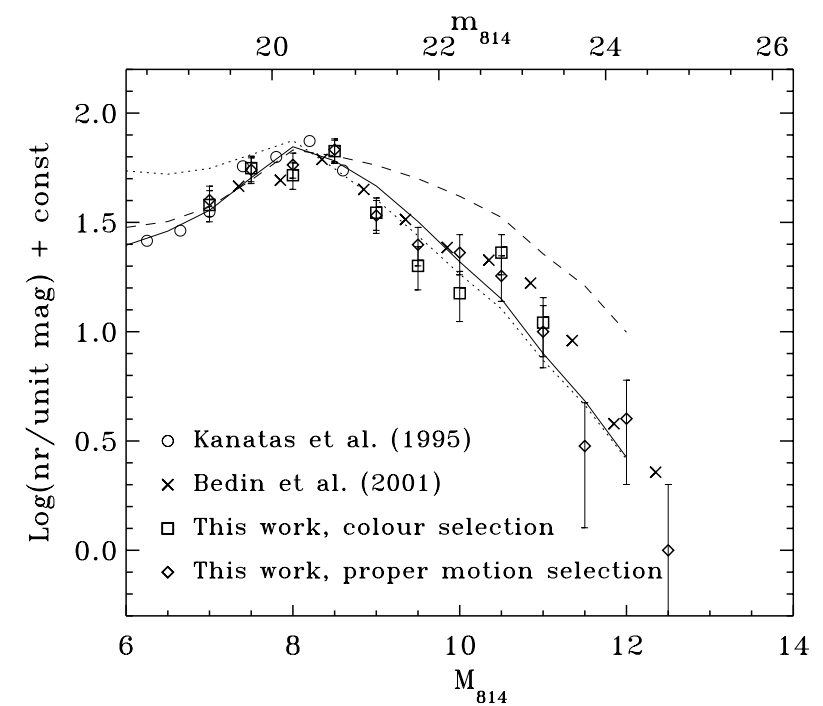

Fig. 3. The data points show the LF of M4 as determined with different methods and by different authors: squares, LF measured from Fig. 2a; diamonds, LF measured from Fig. 2b; crosses, LF as determined by Bedin et al. (2001) from shallower exposures of the same field; plus signs, LF from Kanatas et al. (1995). The lines show various MFs folded through the same M-L relationship (from Baraffe et al. 1997): solid line, TPL with $m_{\mathrm{p}}=0.35 M_{\odot}, \alpha=2.1$ and $\beta=$ 2.7; dashed line, power-law with $\alpha=0.75$; dotted line, power-law with $\alpha=-0.3$.

magnitudes fainter than $m_{814} \simeq 23.5$ the increasing photometric error as well as the paucity of cluster stars in the CMD make it impossible to locate the continuation of the MS amidst field stars. For all practical purposes, then, here the MS becomes 
statistically indistinguishable from field stars and the LF goes to zero.

To overcome this statistical indetermination, we have retrieved from the ESO/ECF archives a set of shallower images of the same cluster region, obtained in 1995 with the WFPC 2 in the F555W and F814W bands, in an attempt to separate cluster and field stars through their proper motion. These data, pertaining to HST programme 5461, are described in Ibata et al. (1999). We have subjected these, first epoch, frames to the same automated calibration and registration procedures used for the deeper, second epoch, exposures described above, forming in this way two combined frames with equivalent exposure time of $29400 \mathrm{~s}$ in F555W and $7200 \mathrm{~s}$ in F814W, respectively. Due to the large proper motion of M4, in the course of the six years between the two epochs cluster objects should have moved, relative to background objects, by about 0 .' 1 , or about a whole WF pixel. To measure this displacement, we have followed the approach outlined in King et al. (1998) and have determined the offset of each star in the reference frame defined by some brighter $\left(19<m_{814}<21\right)$ MS objects in its neighborhood. The latter have initially been selected by virtue of their position in the CMD but, through an iterative procedure, eventually only objects moving less than 0.2 pixel with respect to the average of their peers have been retained as reference MS stars. The coordinates of each object are those of its centroid as determined by the standard IRAF centering procedure (apphot.center). They are very robust since, even near the bottom of the MS, at $m_{814} \simeq 24$, the peak of the typical star is detected at the $\sim 50 \sigma$ and $\sim 20 \sigma$ level above the surrounding background, respectively in the first and second epoch F814W combined frame. Furthermore, at both epochs the combined images are well sampled thanks to the many dithered frames concurring to form them.

Figure $2 b$ shows the CMD of those stars whose position has changed by less than 0.3 pixel between the two epochs (the scatter on the position of the bona-fide MS stars defined above being $\sim 0.1$ pixel). Over-plotted is the same MS band ( $\pm 4 \sigma$ wide) shown in Fig. 2a, which was used to identify obvious outliers not to be included in the counts to derive the LF. The latter is shown as diamonds in Fig. 3, also not corrected for incompleteness, and agrees remarkably well with that determined above (boxes) over the common magnitude range. Below $m_{814} \simeq 23.5$, where the LF derived with the MS-width method goes statistically to zero, there are still a few data points in the CMD (Fig. 2b), which we have assumed to be all MS objects if located more or less along the ideal extension of the ridge line. Considering, however, that at about $1.5 \mathrm{mag}$ brighter there are some objects in the same colour range which proper motion attributes to the cluster but which cannot be considered MS stars, it is fair to wonder whether that assumption is correct. For this reason, we consider the corresponding counts in the LF as upper limits.

Also shown in Fig. 3 is the LF obtained by Bedin et al. (2001) by combining the first epoch data discussed above with some shallower WFPC2 exposures (5300 s long) in the F814W band alone, collected in the year 2000 (crosses). We do not plot their error bars so as not to clutter the graph further, but the agreement is remarkable, thus independently confirming the validity of proper motion studies, even when one of the two epochs only provides information in one band. We note here that this conclusion holds because, in the study of Bedin et al. (2001), the exposure times of the first and second epochs are comparable (namely 7200 s vs. 5300 s). In Sect. 5 we explore the limitations of the proper-motion technique in a rather different scenario.

Deriving the properties of the mass function (MF) underlying the LF shown in Fig. 3 requires the use of a massluminosity (M-L) relationship appropriate for the metallicity of the cluster. As mentioned in Sect. 2 (see Fig. 1), no isochrones today exist that can reproduce the shape of the observed MS in the $V-I, I$ CMD. It is, however, believed (Baraffe et al. 1998; Delfosse et al. 2000; Chabrier 2001) that the $I$ band and near infrared colours are not affected by the same shortcoming in the treatment of the atmospheric opacity mentioned above because the latter is limited to visible colours. Thence, the discrepancy shown in Fig. 1 should not prevent us from obtaining a meaningful MF from the LF measured in the F814W band.

It is, unfortunately, customary to try and fit the observed LF by means of a power-law MF. As Paresce \& De Marchi (2000a) have shown, however, the stellar LF of most GCs measured to date with the HST reveals more structure than that expected if the underlying MF were a simple power-law. According to their analysis of 12 halo GC, the rapid drop of the number counts seen in the LF at $m_{814} \gtrsim 8.5$ is not just the result of the changing source of opacity in the stellar atmosphere (D'Antona 1998) but an inflection in the MF is also needed at $\sim 0.3 M_{\odot}$. Paresce \& De Marchi (2000a) propose that, over the limited mass range covered by GC stars, a log-normal distribution peaked at $\sim 0.3 M_{\odot}$ is more appropriate to describe the MF. Alternatively, De Marchi et al. (2004; see also Paresce \& De Marchi 2000b) propose a tapered power-law (TPL), namely a Salpeter-like distribution which tapers off below a characteristic mass. Analytically, the number of stars per unit mass can be expressed as:

$$
f(m)=\frac{\mathrm{d} N}{\mathrm{~d} m} \propto m^{-\alpha}\left[1-\mathrm{e}^{\left(-m / m_{\mathrm{p}}\right)^{\beta}}\right]
$$

where $m_{\mathrm{p}}$ is the peak mass, $\alpha$ the index of the power-law portion for high masses and $\beta$ the tapering exponent which causes the MF to flatten and drop below the peak mass $m_{\mathrm{p}}$. For the 12 halo GCs studied by Paresce \& De Marchi (2000a), the average values of these parameters are $\alpha=2.3, \beta=2.6$, $m_{\mathrm{p}}=0.35 M_{\odot}$. By folding a function of this type through the M-L relationship of Baraffe et al. (1997) for the metallicity $[\mathrm{M} / \mathrm{H}]=-1$, we are able to reproduce rather well the LF of M 4 over the entire range spanned by the observations with $m_{\mathrm{p}}=0.35 M_{\odot}, \alpha=2.1 \pm 0.1$ and $\beta=2.7 \pm 0.1$ (solid line in Fig. 3). It should be noted that the shape of the LF in the magnitude range only accessible to the proper motion technique does not set any constraints on the value of these parameters, which are already defined by the brighter portion of the LF.

It has been suggested by Richer et al. (2002) that the MF of M4 is consistent with a power-law distribution of index $\alpha=0.75$ over the entire range covered by these observations. A function of this type, folded through the derivative of the 


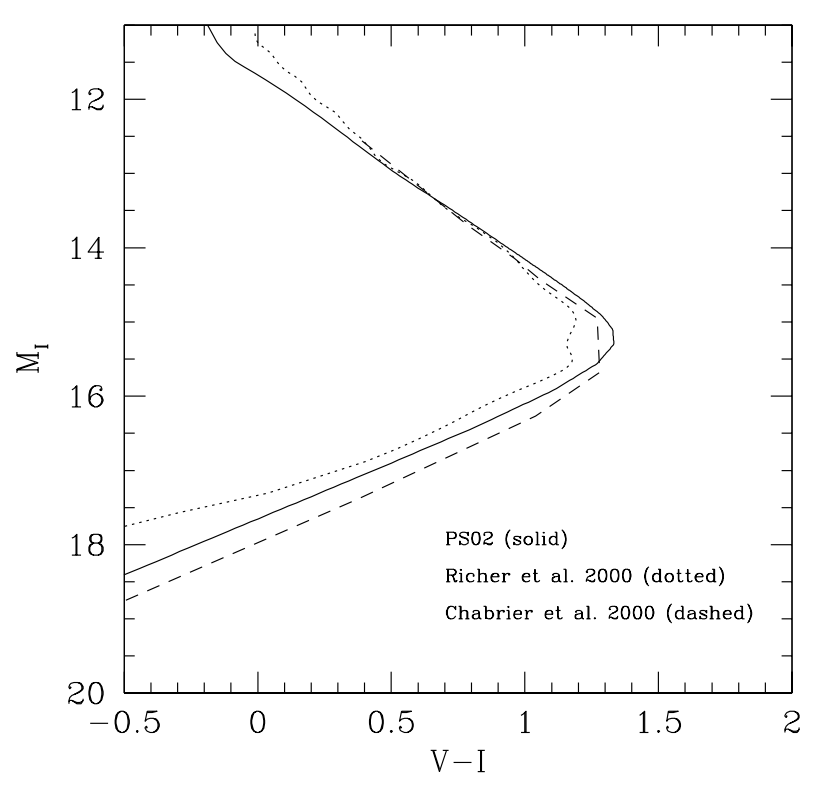

Fig. 4. Evolutionary tracks of a $0.6 M_{\odot}$ WD as predicted by the models of PMS02 (solid line), Richer et al. (2000; dotted line) and Chabrier et al. (2000; dashed line). The filter names $V$ and $I$ here refer to the Johnson/Cousin photometric system.

Baraffe et al.'s (1997) M-L relation, is shown as a dashed line in Fig. 3 and, regardless of the arbitrary registration along the vertical axis, it does not provide a good fit to the data. The dropping portion alone of the LF can be represented with a powerlaw of index $\alpha=-0.3$ (dotted line in Fig. 3). Because of the sign of its index, such a MF increases with mass, thus implying that the number of stars per unit mass does indeed decrease below $\sim 0.3 M_{\odot}$. When this model MF is extended to higher masses, however, it fails to reproduce the $\mathrm{LF}$ at $M_{814} \lesssim 8$. To make this discrepancy more evident, we complement our data at the bright end by showing, as circles, the LF of M 4 as measured by Kanatas et al. (1995) in the $V$ band, after conversion to the $I$ band using the $V, V-I$ CMD of Richer et al. (2002) as a reference.

\section{The white dwarf cooling sequence and the age of the cluster}

Besides the MS, the other notable cluster feature in the CMD of Fig. 1 is the WD cooling sequence, at magnitudes $m_{814} \gtrsim 23$ and colours $0 \lesssim m_{606}-m_{814} \lesssim 2$. The sequence is sparse, yet narrow and well defined down to $m_{814} \simeq 25$, where it broadens because of the increasing photometric uncertainty on the colour, which rapidly grows from $\sim 0.1 \mathrm{mag}$ at $m_{814}=25$ to $\sim 0.4 \mathrm{mag}$ at $m_{814}=27$. The photometric completeness in this region of the CMD decreases accordingly, as indicated above. The sequence tapers off rapidly at $m_{814} \simeq 27$ where the completeness reaches the $50 \%$ level.

A comprehensive analysis of the observed WD cooling sequence requires suitable theoretical models. We base our investigation on the DA WD models recently computed by us (PMS02), which include the latest improvements in the description of the physics of high density matter. As discussed in that paper, theoretical uncertainties are still rather large and they mainly affect the age-luminosity relationship for ages in excess of 6-7 Gyr. These uncertainties should be carefully considered when dating an old WD sequence.

To translate the theoretical luminosity and temperature into magnitude and colour for comparison with the HST observations, we used proper WD model atmospheres computed by Bergeron et al. (2001). An interesting prediction of the theory of the atmospheres of cool WD below $T_{\text {eff }} \simeq 5000 \mathrm{~K}$ is the onset of collision-induced absorption, due to $\mathrm{H}_{2}-\mathrm{H}_{2}$ and $\mathrm{H}_{2}-\mathrm{He}$ collisions at such high densities (Bergeron et al. 1995; Jorgensen et al. 2000). As several authors have shown (Hansen 1998, 1999; Saumon \& Jacobson 1999; Chabrier et al. 2000; Fontaine et al. 2001), the near infrared colours of very cold WDs whose atmosphere contains Hydrogen should be significantly affected by this source of absorption, with the peak of their spectral energy distribution shifting to the blue. A comparison between our adopted evolutionary track of $0.6 M_{\odot}$ and those obtained by Richer et al. (2000) and Chabrier et al. (2000) is shown in Fig. 4 for the Johnson/Cousin photometric system. All three models present an evident turn to the blue ("blue hook") occurring at nearly the same luminosity. The reddest colour is about 0.1 mag bluer for the sequence of Richer et al. (2000). After the "blue hook," the three sequences evolve at different luminosity, with that of Richer et al. (2000) being the brightest and that of Chabrier et al. (2000) the faintest. For example, at $V-I=0.5$ the relative difference in the $I$ band is about half a magnitude. We note here that, by adopting for M 4 the same distance modulus used for MS stars, namely $(m-M)_{I}=12.25$, the discrepancy between these three theoretical cooling sequences is definitely smaller than the photometric errors at the same magnitude level. In other words, the present CMD does not permit a clear discrimination between the different theoretical prescriptions and any conclusion should be independent of the adopted set of WD models. In Sect. 6 we elaborate further on this issue.

The coloured lines over-plotted on the WD sequence in Fig. 1 represent the theoretical isochrones, as calculated from the WD models of PMS02 for ages of 8, 10 and $12 \mathrm{Gyr}$. The initial-final mass relationship and the age of the progenitors have been derived according to the evolutionary models of low and intermediate mass stars with $Z=0.001$ (Dominguez et al. 1999; Straniero et al. 1997), a metallicity in agreement with the latest measurements ${ }^{1}$ of heavy element abundances of giant stars in the field of M4.

The three isochrones overlap one another over most of the magnitude range. In a similar way as for the MS turn-off, the detection of the "blue hook" of the WD cooling sequence in the CMD could provide an estimate of the cluster's age and would represent a much needed sanity check as to the correctness of the models, thus allowing one to rely more heavily on their predictions. Unfortunately, no such feature is visible in Fig. 1 with any statistical significance. To prove this, we show in Fig. 5 an enlargement of the WD region of the CMD of Fig. 1. Although all data points within the indicated

${ }^{1}$ Carretta \& Gratton (1997) find $[\mathrm{Fe} / \mathrm{H}]=-1.19 \pm 0.03$ and Carney (1996) gives $[\alpha / \mathrm{Fe}]=0.3 \pm 0.03$. This would imply $[\mathrm{M} / \mathrm{H}]=0.98$ or $Z=0.0018$. 


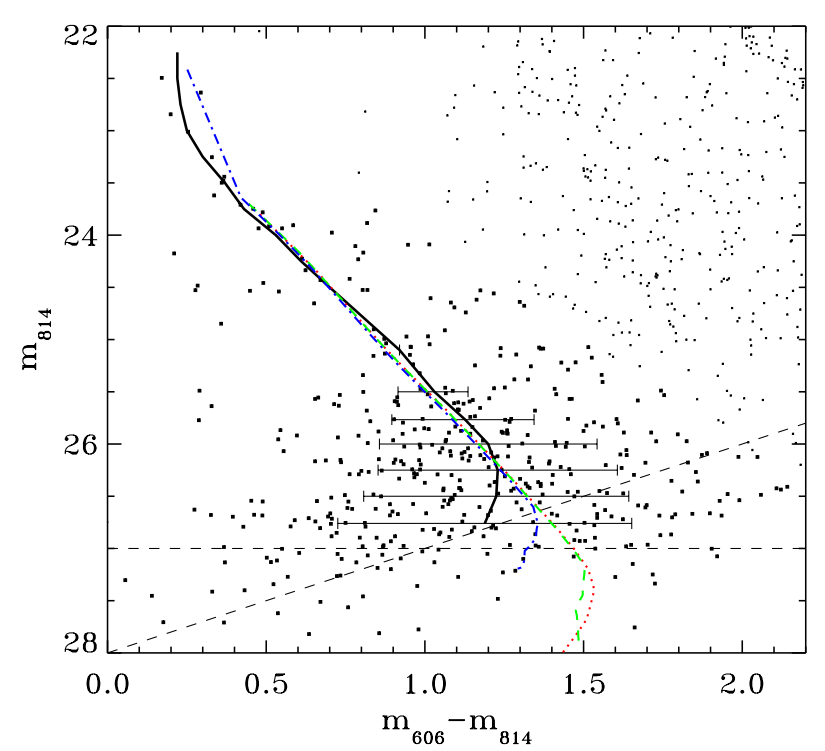

Fig. 5. Enlargement of Fig. 1 showing the region occupied by the WDs. The thick solid line cutting through the data shows the ridge line of the WD cooling sequence estimated as explained in the text. As in Fig. 1, the dashed lines mark the 50\% completeness limits. The WD isochrones for ages of $8 \mathrm{Gyr}$ (blue, dot-dashed), $10 \mathrm{Gyr}$ (green, dashed) and $12 \mathrm{Gyr}$ (red, dotted) are also shown.

colour and magnitude limits are plotted, we only consider here those marked with a stronger print and which comprise the WD region. The thick solid line cutting through the data shows the ridge of the WD cooling sequence as estimated by us. Above $m_{814}=25.5$ this line is simply an eye-ball fit to the points. Below that threshold, however, any evidence of a ridge suddenly vanishes, so there we plot the average colour as a function of the magnitude, surrounded by its standard deviation (the horizontal error bars). Below $m_{814} \simeq 26$, the average colour appears to remain constant or even possibly bluer with increasing magnitude. Given the conspicuous size of the error bars, however, this inflection cannot be interpreted, with any statistical confidence, as the presence of a "blue hook." It could likely suggest a change in the slope of the cooling sequence, but we should point out that an even more likely origin for this effect can be traced in the onset of considerable photometric incompleteness in the F606W band. As in Fig. 1, the dashed lines in Fig. 5 mark the 50\% completeness limits and it is clear that below $m_{814} \simeq 26$ an increasingly larger fraction of redder objects must be missing, thus skewing the distribution to the blue.

If the "blue hook" level is defined as the magnitude of the reddest point in the WD isochrone (in the same way that the MS turn-off is the bluest), for a cluster age of $8 \mathrm{Gyr}$ one should expect to find it at $m_{814} \simeq 26.8$, near the $50 \%$ completeness lines, whereas for 10 and $12 \mathrm{Gyr}$ it should take place at $m_{814} \simeq 27.2$ and $m_{814} \simeq 27.5$, respectively, i.e. more than half a magnitude below our $50 \%$ completeness lines ${ }^{2}$. In any case, because of the large uncertainties in the data, none of the three isochrones (coloured lines in Fig. 5) can be ruled out, nor

\footnotetext{
${ }^{2}$ Our adopted distance modulus, $(m-M)_{814}=12.25$, has been used to translate the isochrones onto the observational plane.
}

can their validity be assessed. Barring the odds that Nature has played tricks on us, i.e. that she has placed the "blue hook" precisely at the detection limit, it would be safer to conclude from this simple test that M 4 must be older than 8 Gyr. As disappointing as it may seem, this is the only sound conclusion that one can infer from the available CMD.

\section{The white dwarf luminosity function and the age of the cluster}

With the above caveat in mind, one could turn to the LF of the WDs for more hints on their age. Several sources of uncertainty come into play here. Firstly, the LF of the cooling sequence depends on assumptions on the relationship between the mass of the progenitors and that of the WD, the time spent by the progenitor on the MS and the initial MF (see e.g. Chabrier 1999). The WD LF is particularly sensitive to the shape of the latter since, although the location of the peak of the WD LF depends only mildly on the slope of the MF (Richer et al. 2000), the rate at which the LF rises to its peak changes dramatically with it (Chabrier 1999), thereby casting serious doubts on conclusions based on a WD LF whose peak is not reached. A sharp maximum is indeed expected in the WD LF of old stellar systems, a characteristic pile up that marks their age (see the recent review by Fontaine et al. 2001). As in the case of the "blue hook," only when the photometry is deep enough to clearly reveal the peak of the LF can one safely constrain the cluster's age. Otherwise, one can only provide a lower limit to it.

Secondly, the WD LF is only meaningful if it is cleaned of as many contaminating field stars as possible. The density of objects seen in the CMD of Fig. 1 between the MS and WD sequence leaves no doubt that the contamination is strong. To clean the WD sequence one should then resort to using the same proper motion technique adopted successfully for MS stars. The problem here is that, the first epoch's exposures being considerably less deep than those of the second, the magnitude range over which one can study the WD LF with some reliability is further reduced. As mentioned above, the dashed lines in Figs. 1 and 5 mark the magnitude at which, on average, the peak of a star on the combined images drops below the level corresponding to 5 times the standard deviation of the surrounding background. If the same criterion were applied to the combined F814W image of the first epoch (the deepest of the two filters), the same $5 \sigma$ line should be drawn at $m_{814} \simeq 25.6$. By relaxing the requirement to just $3 \sigma$ for the first epoch alone, one can lower the limit to $m_{814} \lesssim 26.5$. Below such a threshold, most stars detected in the second epoch do not have a corresponding match in the first one. We show this graphically in Fig. 6, where the same area of the combined F606W+F814W frame is compared at the two epochs, with the same scale of gray levels. Membership can easily be assessed for object No. 1 (a MS star with $m_{814} \simeq 22$ ), No. 4 (a WD with $m_{814} \simeq 25$ ) and No. 5 (a field star with $m_{814} \simeq 25$ ). However, with magnitudes around $m_{814} \simeq 27$, the objects marked Nos. 3 and 4 are well visible at the second epoch (left panel) but they are unmeasurable in the first by any statistically acceptable means at the level of at least $\sim 2 \sigma$ in the peak. 

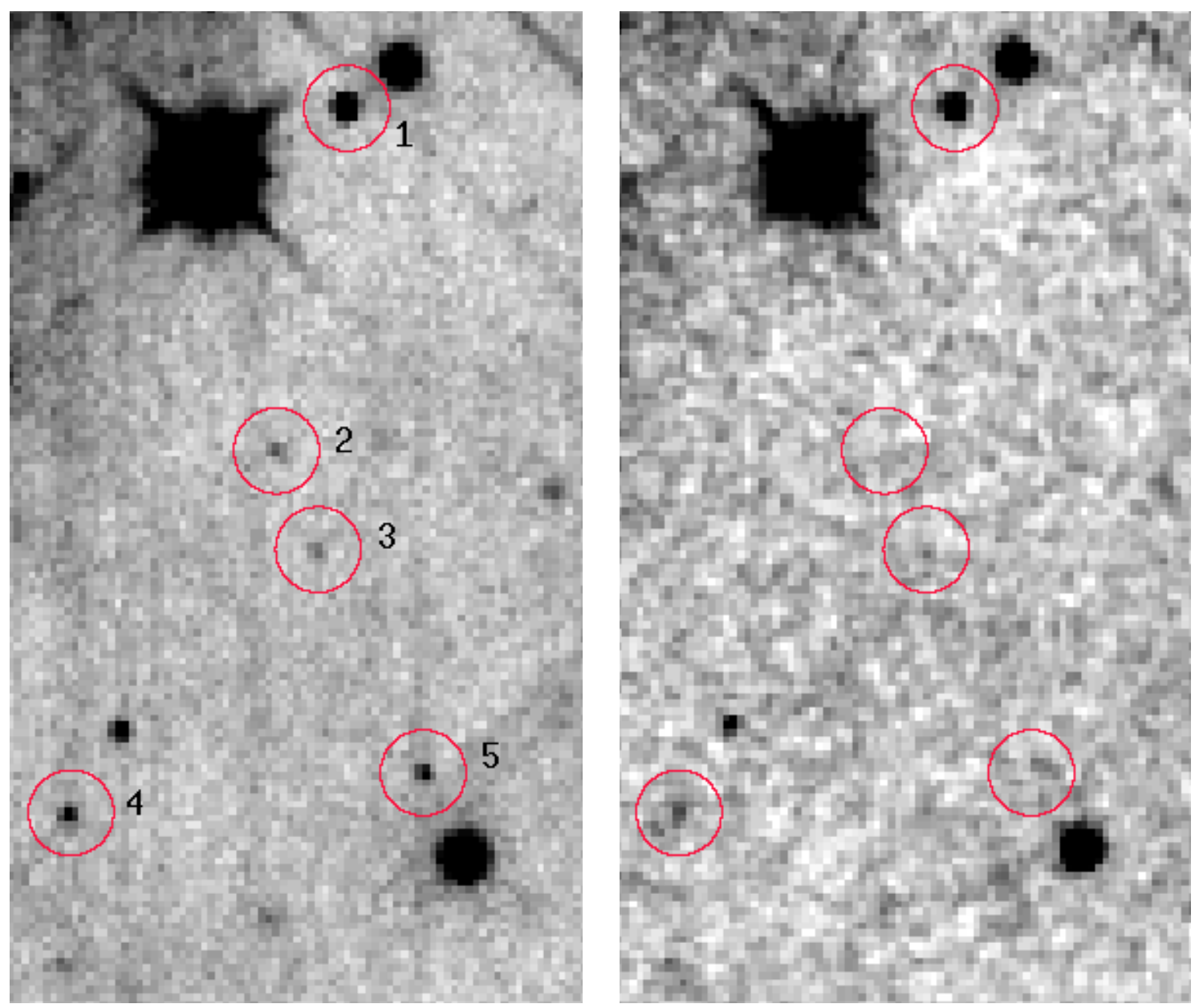

Fig. 6. Left panel: section of $23 \times 38$ square pixel from the WF4 chip from the second epoch (2001). Right panel: same section, but for the first epoch (1995). The scale of grays is the same in both images, which are registered using bona fide MS stars so that no displacement is expected for cluster members. Whilst it is easy to confirm membership for for objects Nos. 1 and 4 and reject it for No. 5, nothing can be said for objects Nos. 2 and 3, both at $m_{814} \simeq 27$, since they are not detected in the first epoch.

Furthermore, since the first epoch combined F814W image (despite being deeper than the F555W one) is the result of only 9 images each of duration $800 \mathrm{~s}$, the background is considerably noisier than in the second epoch F814W frame. It is, thus, possible that one finds there a lump of counts near the position required to match an object in the second epoch, yet there is no guarantee that such a lump represent indeed a true star and, accepting it as such, would require one to make uncertain assumptions about the physical nature of the sources detected in the deeper exposure, thus invalidating the basis on which proper motion studies rest. For this reason, we believe that it is safer to consider as valid only stars which can be detected as such (at least at the $3 \sigma$ level) in the first epoch.

In Fig. 7 we show the CMD of the objects in the WD region. Solid points mark all the stars detected in the second epoch (the same as Fig. 5), whereas the squares indicate the objects which were found in the first epoch as well and whose displacement is smaller than 0.3 pixel with respect to the nearby bona fide MS stars. (This is the same selection criterion adopted for Fig. 2). The thick solid line cutting through the WD sequence is the ridge line, determined as in Fig. 5. Although the ridge line begins to steepen at $m_{814} \gtrsim 25.5$, its shape is fully compatible with that of Fig. 5 (thin solid line) within the errors. Consequently, not even in this case (i.e. by using only the bona fide cluster stars) is it possible to discriminate amongst the isochrones corresponding to the three ages mentioned above. The broadening of the cooling sequence with increasing magnitude, witnessed by the growing size of the error bars, is totally compatible with the photometric error. Thus, within such an uncertainty, there is once again no indication of a turn to the blue. To measure the WD LF, we counted the number of objects in Fig. 7 which are within $4 \sigma$ of the ridge line, where $\sigma$ is the photometric uncertainty on the colour indicated on the lefthand side of the graph. The LF so derived is listed in Table 1.

For an improved comparison between theory and observations, we have produced a set of synthetic CMDs and LFs for WD stars, as explained in the following. Firstly, we assume an age and the index of a power-law MF $(\alpha)$ for the cluster. The minimum mass of the WD progenitors depends on the assumed age, whereas the maximum mass is fixed according to the detection limit, namely $m_{814}=27$ for the available photometry. We, therefore, use a Monte Carlo method to generate values of the progenitor's initial mass, distributed according to the assumed MF. The relationship between initial and final mass then 


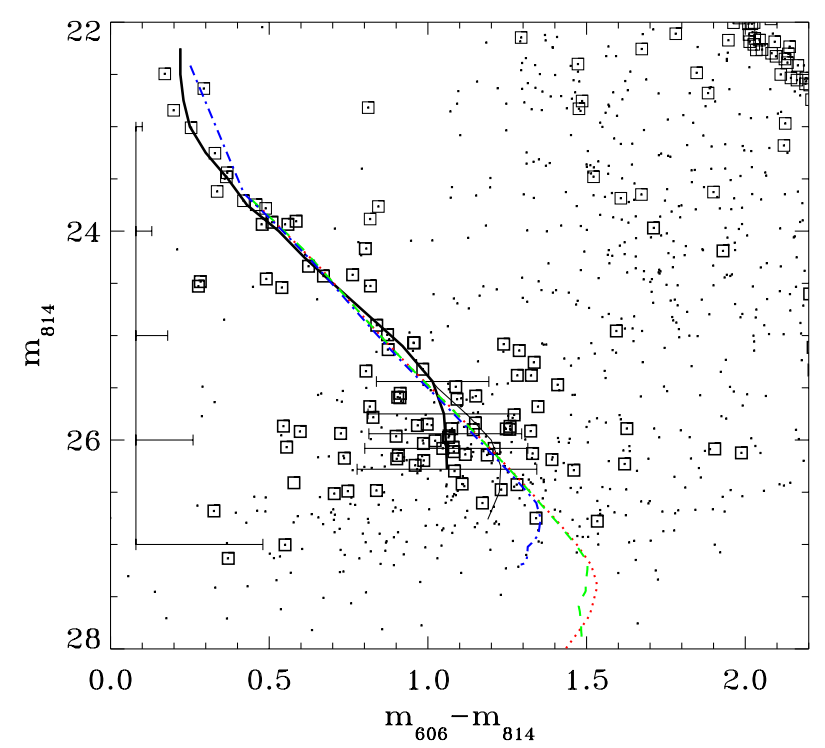

Fig. 7. Colour-magnitude diagram of the region occupied by the WDs. Solid dots indicate all stars from Fig. 5, whereas open squares mark the objects that moved less than 0.03 (i.e. $0.3 \mathrm{WF}$ pixel) between the two epochs. The thick solid line, showing the WD ridge line determined as explained in the text, is here compared with the ridge line of Fig. 5 (thin solid line). The coloured lines are the same WD isochrones shown in Figs. 1 and 5 for ages of $8 \mathrm{Gyr}$ (blue, dot-dashed), $10 \mathrm{Gyr}$ (green, dashed) and $12 \mathrm{Gyr}$ (red, dotted).

Table 1. White dwarf luminosity function. Column (1) gives the $M_{814}$ magnitude at the centre of each bin, Col. (2) the number $N_{0}$ of WDs counted in that bin and Col. (3) the associated photometric completeness.

\begin{tabular}{crc}
\hline \hline$M_{814}$ & $N_{0}$ & compl. \\
\hline 10.0 & 1 & 1.00 \\
10.5 & 2 & 1.00 \\
11.0 & 5 & 0.95 \\
11.5 & 7 & 0.95 \\
12.0 & 5 & 0.95 \\
12.5 & 4 & 0.95 \\
13.0 & 13 & 0.80 \\
13.5 & 24 & 0.80 \\
14.0 & 27 & 0.65 \\
\hline
\end{tabular}

gives the mass of the corresponding WD, whose location in the CMD is obtained by interpolating on the grid of models of PMS02. Finally, photometric errors are applied according to a Gaussian distribution, similar to the one observed. The extraction of the MS mass values is iterated until the number of WDs having $m_{814}<26.5$, or $M_{814}<14.25$ with our adopted distance modulus, matches the one inferred from the observed CMD after correction for incompleteness, namely 112 . An example of such a synthetic CMD is shown in Fig. 8, for an age of $12 \mathrm{Gyr}$ and $\alpha=2.3$. Synthetic LFs are shown for various values of $\alpha$ in Fig. 9, and for different ages in Fig. 10. The agreement with the measured LF (open squares) is excellent, in all cases except 8 Gyr (see Fig. 10).

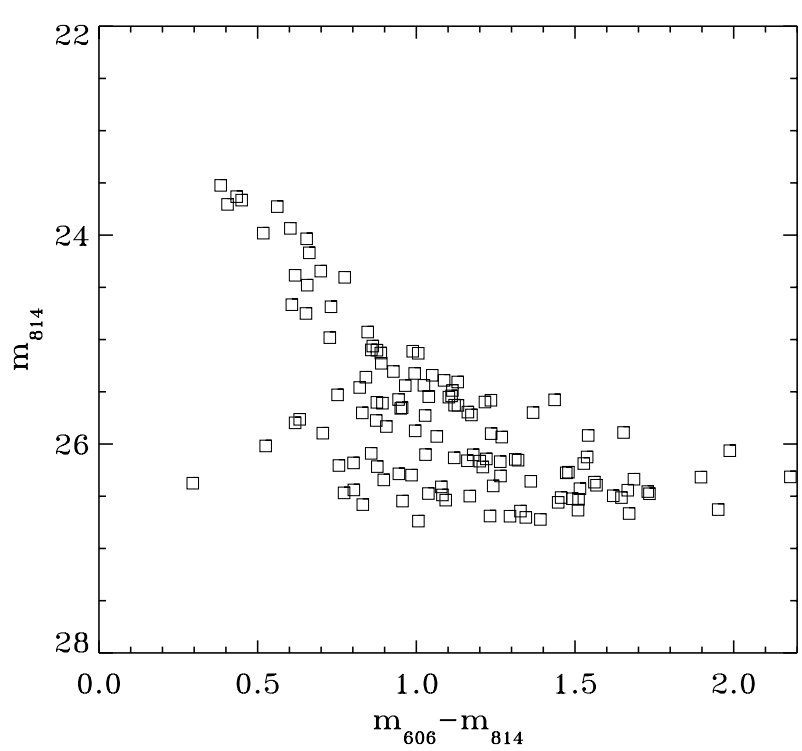

Fig. 8. Example of a synthetic CMD, based on the models of PMS02, for the WDs in a GC of 12 Gyr of age and initial MF index $\alpha=2.3$. This CMD, which does not include contamination from field stars and is photometrically complete at all magnitudes, can be compared with that of Fig. 7.

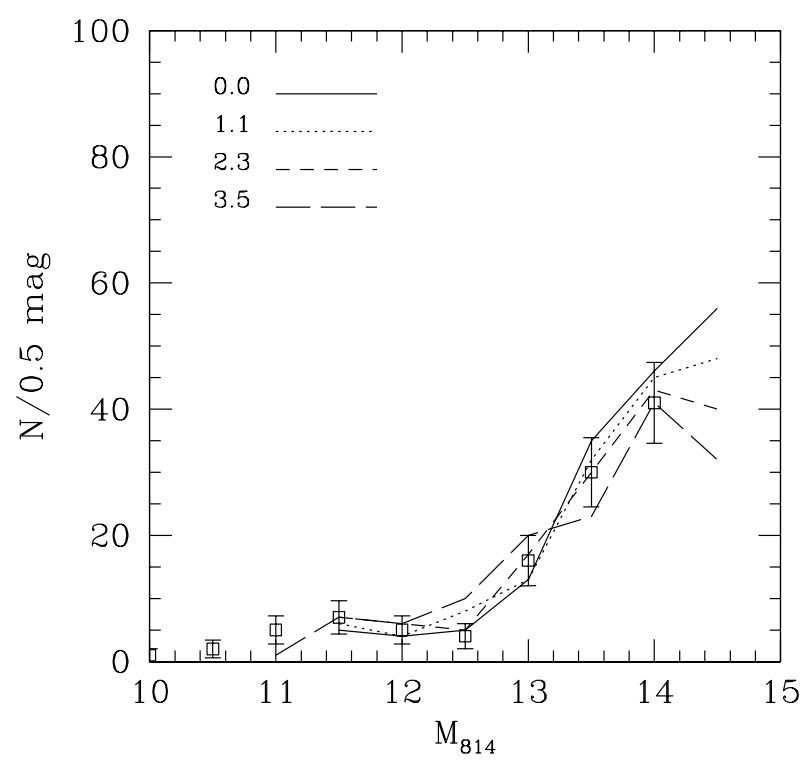

Fig. 9. Comparison between the empirical WD LF of Table 1 (squares) and those predicted by our models for $12 \mathrm{Gyr}$ of age. Each line corresponds to a different index of the initial MF, as indicated in the legend. All models fit the data remarkably well.

The good match between theory and observation provides an important confirmation of the validity of the theoretical models of the WD cooling sequence at least up to $M_{814} \simeq 14$. The brightest and the faintest WD in the synthetic CMD shown in Fig. 8 have a model mass of 0.5566 and $0.5901 M_{\odot}$, respectively, which correspond to progenitor masses of 0.8660 and $1.2028 M_{\odot}$ on the MS, respectively. A look at the internal structure of the models close to the faint end of the observed sequence also provides interesting information: the oxygen component of the core is almost fully crystallised, whilst 


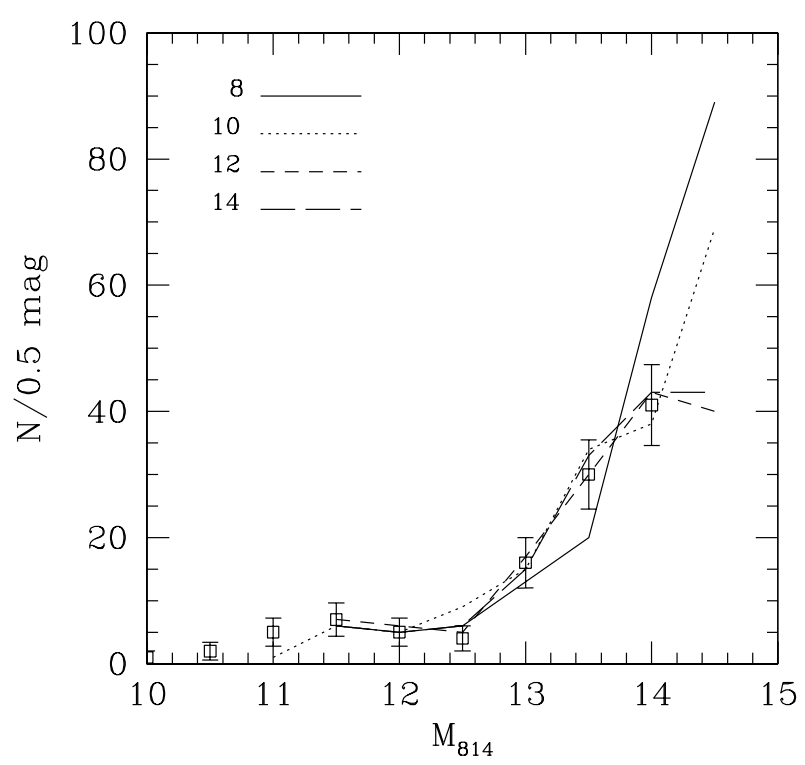

Fig. 10. Comparison between the empirical WD LF of Table 1 (squares) and those predicted by our models for an initial MF index $\alpha=2.3$. Each line marks a different cluster age, as indicated in the legend in Gyr. The models with an age in excess of $8 \mathrm{Gyr}$ fit the data remarkably well.

only $20-25 \%$ (in mass) of the carbon is in the solid phase (the rest being liquid). Furthermore, for the faintest WDs observed (at $M_{814} \simeq 14$ ), the so called "convective coupling" (see e.g. Fontaine et al. 2001) has just begun. This phenomenon occurs when the base of the convective envelope reaches the inner regions of the star, where heat transport is dominated by electronic conduction. We recall that both the release of latent heat caused by the liquid/solid phase transition and the convective coupling induce a substantial decrease of the cooling timescale and, in turn, affect the age-luminosity relationship. This delay of the cooling is responsible for the expected pile up of white dwarfs. Figures 9 and 10 show that the rate of growth of the observed number of WDs brighter than $m_{814} \simeq 26.5$ $\left(M_{814} \simeq 14.25\right)$ does not depend on the initial MF nor on the age. Therefore, the very good agreement between theoretical and observed LF down to the detection limit in M4 strongly supports our description of the complex physical phenomena operating in extreme conditions (high pressure and low temperature).

Unfortunately, in spite of this confirmation, little can be concluded from this data set about the cluster properties such as its age and initial MF. In fact, it is immediately evident that, within the uncertainties, all ages displayed in Fig. 10 are compatible with the data, although for an age of 8 Gyr a very steep initial MF $(\alpha>3.5)$ would be required to reproduce the WD number counts in the faintest magnitude bin. Thus, one may tentatively conclude that M4 is older than 9-10 Gyr (although also these values are model-dependent), thence placing a slightly improved lower limit with respect to that already derived by means of the WD isochrone fitting method. No evidence of a maximum of the LF is obtained and, in turn, no upper limit for the age of M 4 can be set on the basis of these data.

\section{Comparison with previous analyses}

As mentioned in the Introduction, a previous analysis of these same data by Richer et al. (2002) arrived at the rather different result that the age of M 4 is $12.7 \pm 0.7$ Gyr (Hansen et al. 2002). Although we are not able to reconcile completely our result with theirs, we offer here some considerations that might indicate where the origin of this discrepancy lies.

The first notable difference is in the assumed distance modulus for M4, which we took from Harris (1996) to be $(m-M)_{V}=12.83$ with colour excess $E(B-V)=0.36$, which in turn imply $(m-M)_{I}=12.25$. The other widely used catalogue of GC parameters, that of Djorgovski (1993), gives $(m-M)_{V}=$ 12.75 and $A_{V}=1.24$, which translate into $(m-M)_{I}=12.11$. Hansen et al. (2002) used $(m-M)_{V}=12.51$ based on MS fitting with subdwarfs of known parallax (see Richer et al. 1997). The application of such a subdwarf-fitting method requires great caution. Amongst the many sources of uncertainty, the most severe is related to the colour shifts that must be applied owing to the differences in reddening and metallicity between cluster and subdwarf stars (see e.g. Gratton et al. 1997; Pont et al. 1998; Carretta et al. 2000; Gratton et al. 2003). For example, an error in the global cluster metallicity $([\mathrm{M} / \mathrm{H}])$ of just 0.1 dex implies a change of the estimated distance modulus of about $0.1 \mathrm{mag}$. In this context, the metallicity adopted by Richer et al. (1997) in their application of the subdwarf fitting method, namely $[\mathrm{Fe} / \mathrm{H}]=-1.3$ without any account for $\alpha$-elements enhancement, is at odds with the $[\mathrm{M} / \mathrm{H}]=-1$ value implied by the high resolution spectroscopy quoted above (Carretta \& Gratton 1997; Carney 1996). By adopting this latter value for the global metallicity of M 4, the subdwarf fitting method would give a distance modulus of $(m-M)_{V}=12.86$, perfectly in line with the one that we assumed.

The second difference is the adopted photometric system. We simply calibrated our instrumental magnitudes in the HST in-flight system (VEGAMAG), which only requires the application of a zero point. Richer et al. (2002), however, translated their photometry into the Johnson/Cousin ground-based system and notice a large colour term in the conversion from F606W to $V$. Thus, it cannot be excluded that, there not being observed spectra for WDs of the type studied here, the uncertainty in the conversion is very large. On the other hand, we used for the WDs the models of PMS02, which were generated in the same VEGAMAG system by using the atmospheres of Bergeron et al. (2001), whilst Hansen et al. (2002) used the models computed by Hansen (1999) for the Johnson/Cousin system.

In order to make it possible to compare our results with those of Hansen et al. (2002), we measured the WD LF in F606W and attempted to convert it into Johnson's $V$ band. There not being in these bands observations of spectrophotometric WDs with the mass, gravity and age of relevance here, we were forced to base this translation on the theoretical WD isochrones of PMS02, which, besides mass and gravity, provide the magnitudes of the cooling WDs in various bands of the VEGAMAG and Johnson's photometric systems. It is, thus, possible to derive the $V$ magnitude of each bin of the WD LF directly from the mass corresponding to the observed 


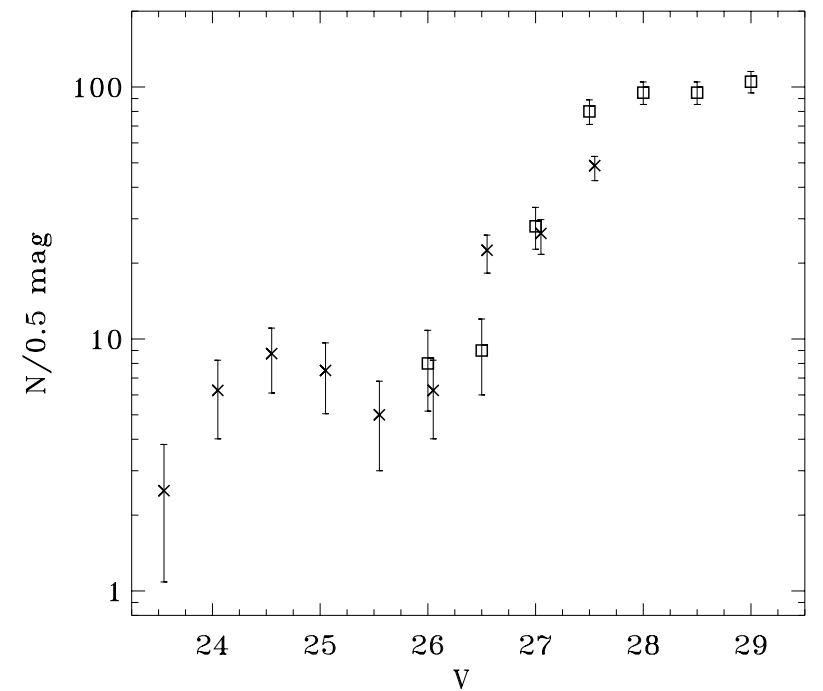

Fig. 11. The WD LF as measured by Hansen et al. (2002) in the $V$ band (squares) is compared here with ours (crosses).

F606W magnitude. Given the remarkably good agreement between the observed and predicted WD cooling sequence (see Fig. 1) as well as the fact that the F606W and $V$ band span a very similar wavelength range, the colour term is practically negligible. The $V$-band WD LF so obtained is shown in Fig. 11 (crosses), where it can be directly compared with that published by Hansen et al. (2002, squares). Both LFs are corrected for photometric incompleteness (never exceeding 50\% for ours). As expected, since our proper motion selection is more conservative than that of Hansen et al. (2002; see Fig. 5), our $V$-band LF does not reach as deep as theirs. The comparison is further hampered by the fact that Hansen et al. (2002) do not show the LF for $V<26$. Over the common magnitude range, however, the two LFs agree to within $\sim 2 \sigma$. We underline here, however, that given the uncertainties of the conversion from F606W to $V$, Fig. 11 is only meant to show the comparison between our LF and that of Hansen et al. (2002). The most reliable LF still remains that in the F814W band.

Besides directly comparing the two $V$-band LFs, it is also possible to compare the WD LF as predicted by our models (PMS02) in that band with the measurements of Hansen et al. (2002). We do so in Fig. 12, where the squares indicate the WD LF of M4 as measured by Hansen et al. (2002), corrected for incompleteness. We note that the theoretical WD LF of the same authors reproduces the observations stunningly well for an age of $12.5 \mathrm{Gyr}$, a distance modulus $(m-M)_{V}=12.51$ and a power-law initial MF of intermediate index $(\alpha \simeq 1)$. With the same distance modulus and MF index, the LF predicted by PMS02 departs significantly from the observations, regardless of the adopted age. In particular, we are unable to reproduce the ratio between the bright and the faint end of the WD LF as derived by Hansen et al. (2002). To reduce the discrepancy, we should be forced to use an unphysically flat MF $(\alpha=0)$ which, however, still gives a rather unsatisfactory fit even for an age of $13 \mathrm{Gyr}$ (see dotted line in Fig. 12). However, it did not escape our attention that the contamination from non cluster members at the faint end of the LF (not unlikely, given

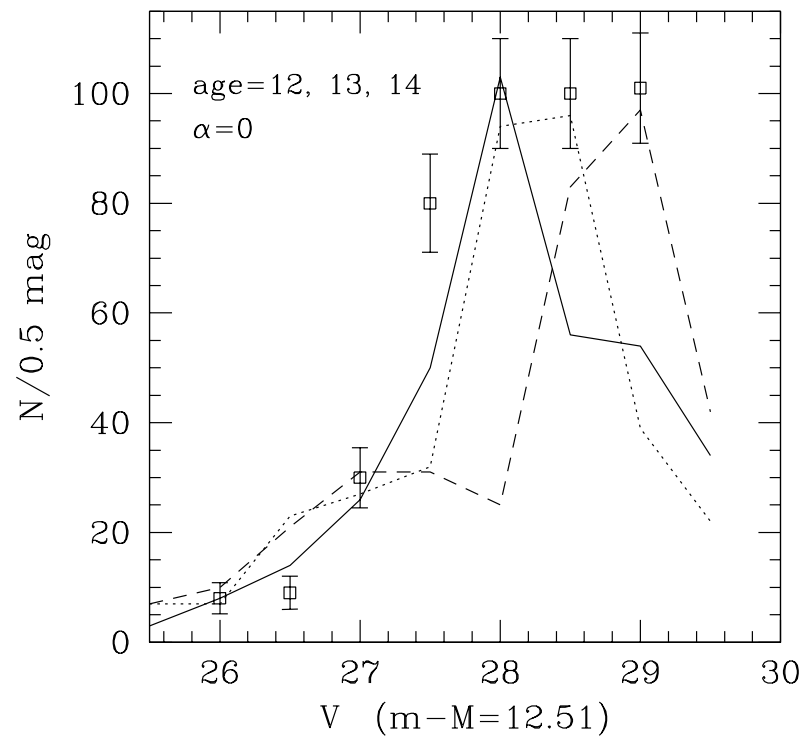

Fig. 12. The WD LF as measured by Hansen et al. (2002) in the $V$ band (squares) is compared here with our model LFs in the same band, which are evidently unable to reproduce the observations. The least unsatisfactory fit (dotted line) is obtained for an age of $13 \mathrm{Gyr}$ but requires an unphysically flat initial MF $(\alpha=0)$.

the conditions under which Hansen et al. 2002 have assessed cluster membership for the faintest objects; see Sect. 5) may indeed mimic the effect of a flat initial MF. In fact, the photometric completeness of the data-point at $V=29$ does not reach $40 \%$.

A disagreement is, thus, evident between the two sets of theoretical LFs, which, however, does not correspond to a difference in the cooling sequences, as witnessed by Fig. 4. To investigate further the origin of this discrepancy, we have compared the theoretical WD LFs of PMS02 with those tabulated of Richer et al. (2000), which are based on the models of Hansen (1999). An example is shown in Fig. 13 for an age of $12 \mathrm{Gyr}, \alpha=2.35$ and progenitors of solar composition: although the peaks of the two LFs are displaced by $\sim 0.5 \mathrm{mag}$, the rise and drop are quite similar. In particular, no plateau is seen at the faint end such as the one obtained by Hansen et al. (2002). Thus, it seems that the difference between our fit to the WD LF of M4 and that of Hansen et al. (2002) cannot be attributed to the different theoretical prescriptions for the physics of the cooling process. We can only speculate that the discrepancy could be due to differences in the adopted progenitor ages and/or in the initial-final mass relationship.

Nevertheless, a general comment on the comparison between theory and observation can still be done: since the number of WDs is still rising in the faintest available bin of the observed LF (both in ours in the F814W band and in that of Hansen et al. in the $V$ band), only a lower limit to the age can be derived. Once again we emphasise that a clear identification of the WD LF peak is a mandatory requirement for a reliable determination of the cluster's age.

Deeper observations, with the sensitivity and accuracy now attainable with the Advanced Camera for Surveys (ACS) on board the HST would dramatically improve this situation. 


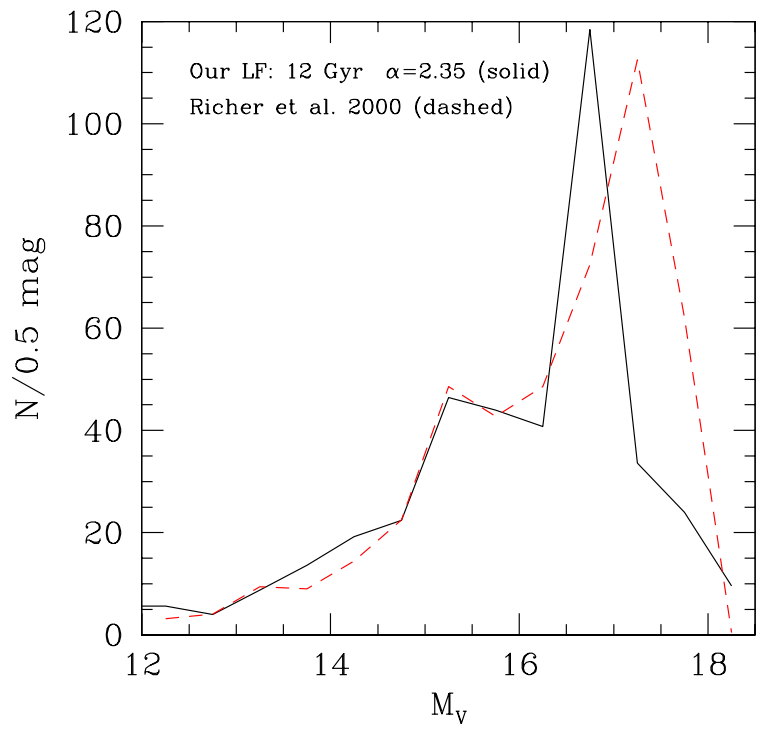

Fig. 13. Theoretical WD LFs for cluster age of $12 \mathrm{Gyr}$ and initial MF index $\alpha=2.35$ as predicted by our models (PMS02; solid line) and by those of Richer et al. (2000; dashed line). Both models predict a sharp LF peak, rather different from the plateau measured by Hansen et al. (2002).

Synthetic CMDs provide us with a powerful tool to check the effectiveness of measurements at fainter limits. In Fig. 14 we show a synthetic CMD in which the detection limits have been moved down to $m_{814} \simeq 30$. Photometric errors have been re-scaled in such a way that they coincide, at the faint limit, with the photometric errors of the present photometry. As expected, for the adopted age of 13 Gyr and $\alpha=2.3$, a clump around $m_{814}=28$ appears. At fainter magnitudes, owing to both the steepness of the initial MF and the acceleration of the cooling time, only few stars are predicted, scattered by the large photometric errors. Since the field of view of the ACS is about 2.3 times larger than that of the WFPC2, we have supposed that 260 WDs (instead of 112) should be found for $m_{814}<26.5$. Figure 15 illustrates the expected variation of the LF for different ages, after proper normalisation to the total number of stars observed. Interestingly, the peak of the WD LF is extremely sensitive to the age, shifting at a rate of $\sim 0.5 \mathrm{mag} / \mathrm{Gyr}$ (for ages ranging between 10 and $13 \mathrm{Gyr}$ ), whereas the corresponding variation of the MS turn-off luminosity is only $\sim 0.1 \mathrm{mag} / \mathrm{Gyr}$ (for an age of $12 \mathrm{Gyr}$ ). This implies that the age estimated by means of the WD LF is considerably less sensitive to the uncertainty on the distance than the latter. On the basis of these simulations, we conclude that the peak of the WD LF in M4 should be clearly identifiable by means of the ACS in a reasonable exposure time, if the cluster's age is $<13.5 \mathrm{Gyr}$ as expected from the recent WMAP measurements.

\section{Summary}

The main results of this paper can be summarised as follows.

1. We have reduced and analysed a set of deep observations of the GC M 4 obtained in 2001 with the WFPC2 camera on board the HST. This data set includes 98 images in the F606W filter and 148 images in the F814W band, each of duration $1300 \mathrm{~s}$. All the frames have been subjected to the standard HST pipeline which resulted in two calibrated, registered and co-added images (one per filter) on which standard aperture photometry was run. We show that stars can be reliably measured down to the $50 \%$ completeness limit at $m_{606} \simeq 28, m_{814} \simeq 27$. Above magnitudes brighter than $m_{814} \simeq 19$ the photometry is not reliable because of saturation.

2. We derive a CMD (Fig. 1) which reveals a narrow and well defined stellar MS extending to $m_{814} \simeq 23$, where it becomes indistinguishable from field stars. The WD cooling sequence is also visible and extends from $m_{814} \simeq 22.5$ through to the detection limit where it broadens considerably due to the increasing photometric uncertainty and where field star contamination is most severe. The signature of the Galactic disc and bulge is clearly visible in the CMD as a cloud of points occupying the region between the WD cooling sequence and the MS.

3. The shape of the cluster MS differs from that predicted by the models of Baraffe et al. (1997) for any choice of distance modulus. This shortcoming in the theoretical description of low-mass stars has already been noted by Bedin et al. (2001) and stems from the inadequacy of the available treatment of the $\mathrm{TiO}$ molecule opacity (Chabrier 2001) in the F606W and bluer bands. We derive the LF of MS stars in the F814W band, where uncertainties in model atmospheres are smaller, by imposing a colour selection around the MS ridge line on the CMD down to $m_{814}=23.5$ or $M_{814}=11$ (Fig. 2). We show that the LF is not compatible with a single power-law underlying MF, regardless of the adopted index. Instead, the observations are well reproduced by a tapered power-law MF with peak mass $m_{\mathrm{p}}=0.35 M_{\odot}$, index $\alpha=2.1$ and tapering exponent $\beta=2.7$ (Fig. 3). For comparison, the average parameters for 12 halo GCs studied with the HST are $m_{\mathrm{p}}=0.34$, $\alpha=2.3, \beta=2.6$.

4. Adopting the canonical distance modulus for M 4 of ( $m-$ $M)_{I}=12.25$, our theoretical WD isochrones overlap remarkably well with the observed cooling sequence (Fig. 5). The data, however, do not reach the magnitude domain where isochrones of different ages depart significantly from one another, nor that where the rapid turn to the blue of the sequences ("blue hook") caused by collision-induced absorption is expected to take place. Therefore, only a lower limit of $\gtrsim 8 \mathrm{Gyr}$ can be set to the age of M 4 from the CMD alone.

5. To set more stringent constraints on cluster membership than those allowed by the colour of the stars in the CMD and, thus, derive accurate LFs for both MS stars and WDs, we have reduced and analysed a set of shallower observations of the same cluster field obtained in 1995 with the WFPC2 with the aim of measuring proper motions. The considerably shorter exposure times (particularly in the F814W band) forced us to restrict this study to objects brighter than $m_{814} \simeq 26.5$, since fainter objects are not detectable by any statistical means at the level of at least $3 \sigma$ 

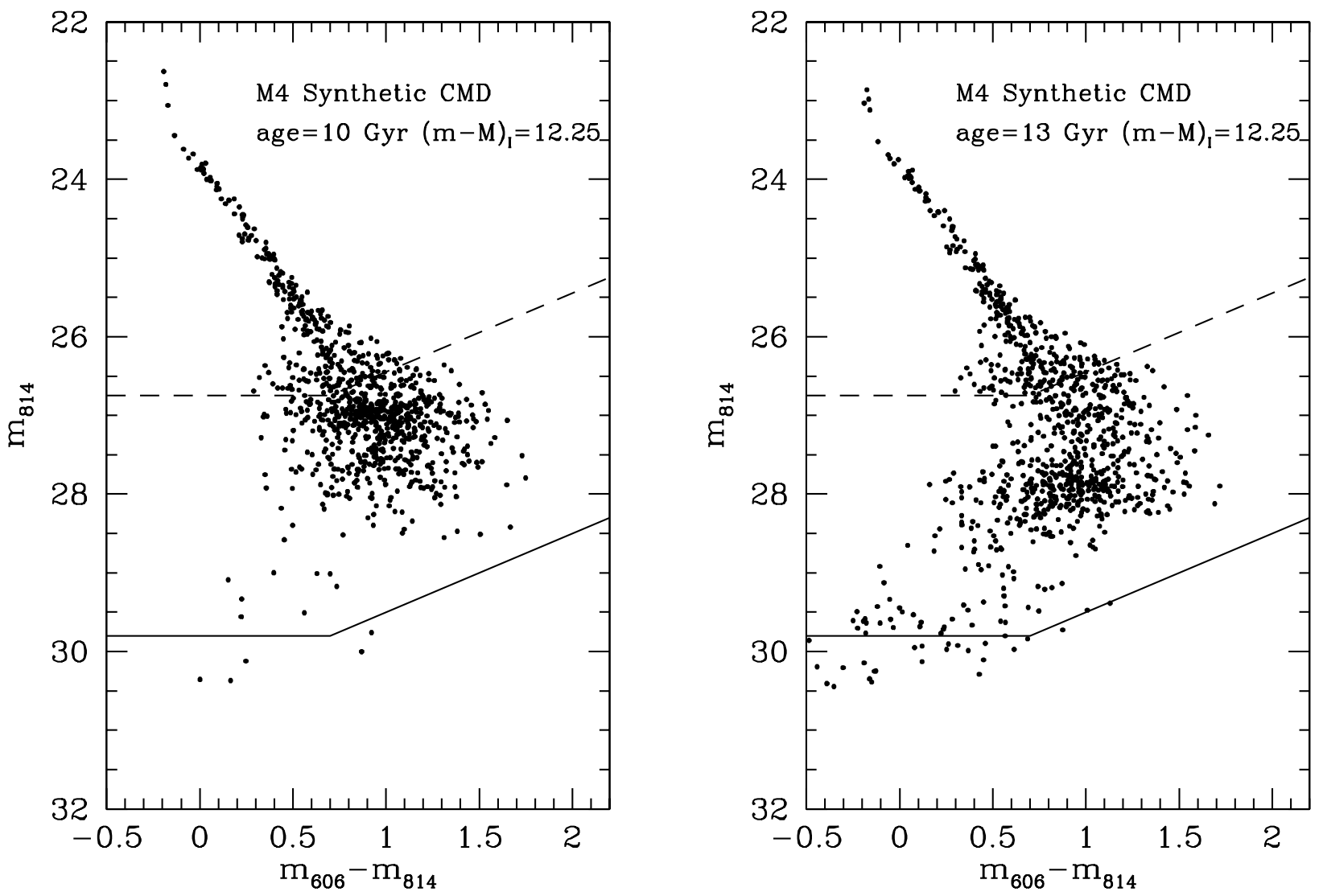

Fig. 14. Synthetic CMDs simulating the WD cooling sequence in M4, as it would be observed with the ACS on board the HST, for a cluster age of $10 \mathrm{Gyr}$ (left-hand panel) and $13 \mathrm{Gyr}$ (right-hand panel). The "clump" of stars responsible for the peak of the WD LF moves to fainter magnitudes with increasing age.
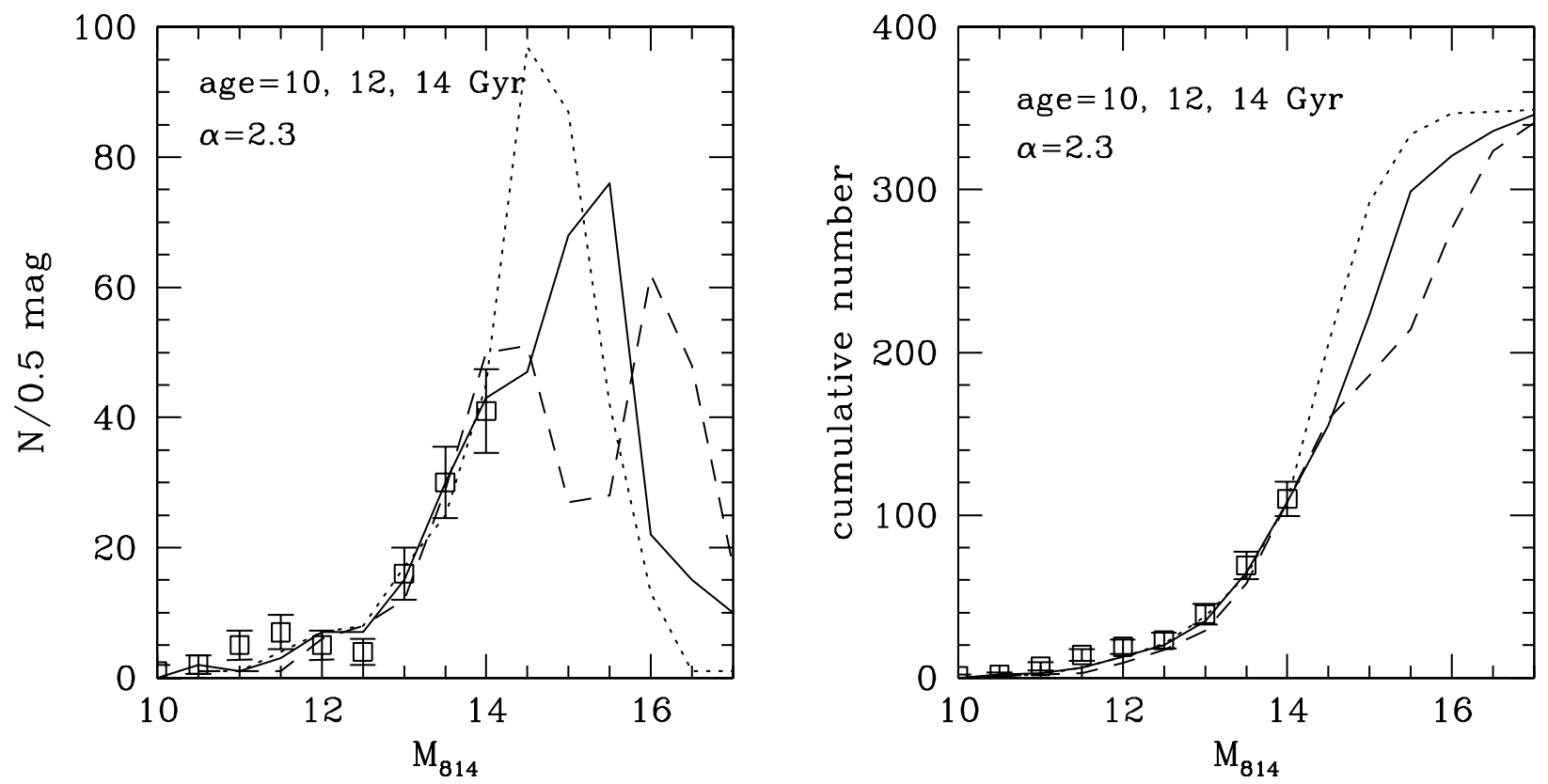

Fig. 15. The sensitivity of the ACS on board the HST makes it possible to access the magnitude range above the limit currently set by the WFPC2 observations (squares) at $M_{814} \simeq 14$, where the WD LF is most sensitive to the cluster's age. Theoretical differential and cumulative WD LFs are shown, respectively in the left- and right-hand side panel, for an initial MF index $\alpha=2.3$ and the ages as indicated in the legend. 
and, therefore, their presence, position and nature cannot be securely confirmed (Fig. 6). Above this limit, cluster members can rather easily be separated from field stars since their displacement amounts to $\sim 0{ }^{\prime} 1$ between the two epochs, or a full WF pixel.

6. The LF of MS stars, selected this time via their proper motions, agrees remarkably well with that obtained through colour selection in the CMD over the common magnitude range. The former, however, extends further to $m_{814}=25$ or $M_{814}=12.25$ where the number of MS star is consistent with being zero. The underlying MF that best fits this LF is the same tapered power-law distribution mentioned above, thus suggesting that, although important for obtaining information on the faint MS end, the availability of proper motions does not substantially improve our understanding of the number and distribution of low mass stars. In fact, we stress that the known shortcomings in modelling the atmosphere of very low mass objects (see Fig. 1) make it difficult to decide whether and where one has reached the bottom of the MS.

7. On the other hand, knowing which objects belong to M4 makes it possible to study the LF of the WDs, whose location in the CMD is severely contaminated by field stars. However, the shallower photometric depth of the first epoch's data limits this investigation to $M_{814}<14.5$, thereby preventing us from exploring the domain where the WD LF is most sensitive to the cluster's age and to its initial MF. Our models show that MF indices in the range $0<\alpha<3.5$ and ages in the range 8-14 Gyr are all consistent with the data (Figs. 9 and 10), although for an age of 8 Gyr an unrealistically steep MF ( $\alpha>3.5)$ would be required to reproduce the number counts in the faintest bin of the LF. On the basis of these data and our models, we can set a lower limit of $\gtrsim 9$ Gyr to the age of M4. We underline here that an upper limit to the age can only be set when the sharp maximum of the WD LF is detected which results from the characteristic pile up of old WDs along their cooling sequence (Fontaine et al. 2001). No such feature is seen in the presently available data and, therefore, only a lower limit to the age can be set.

8. We have compared our results with those of Hansen et al. (2002) who, using the same data set, derived for M 4 an age of $12.7 \pm 0.7 \mathrm{Gyr}$ from the WD LF in the $V$ band. We are unable to reproduce satisfactorily their observations with our theoretical $V$-band LF for any physically meaningful value of the WD progenitors' initial MF index $\alpha$. In particular, we are not aware of models predicting a plateau in the LF such as the one obtained by Hansen et al. (2002) for $V \gtrsim 28$. The latter could be the consequence of an excess of spurious WDs at the faint end of the LF arising from uncertainties in the proper motions of objects which are difficult to detect in the first epoch. Nevertheless, since the WD LF of Hansen et al. (2002) is still rising at the faintest bin, no upper limit to the age can anyhow be set.

9. We have used synthetic CMDs to simulate deeper GC observations such as those today attainable with the ACS on board the HST. The combination of a wide field of view and excellent sensitivity in the F606W and F814W bands makes it possible for this instrument to study the physical properties of old WDs, to detect the "blue hook" in their cooling sequences and to locate with accuracy the peak of their LF, thereby determining their age, if M 4 is younger than $\sim 13.5$ Gyr as the recent WMAP measurements indicate. Since the peak of the LF moves by 0.5 mag per Gyr of age (for ages ranging between 10 and 13 Gyr; Fig. 15), the latter can be measured with an accuracy comparable with that of $H_{0}$, thus setting robust cosmological constraints to the time of GC formation.

Acknowledgements. In this work we have made extensive use of the ESO/ST-ECF archival facilities. It is our pleasure to thank an anonymous referee for providing us with comments that have considerably strengthened the presentation of this work.

\section{References}

Baraffe, I., Chabrier, G., Allard, F., \& Hauschildt, P. 1997, A\&A, 327, 1054

Baraffe, I., Chabrier, G., Allard, F., \& Hauschildt, P. 1998, A\&A, 337, 403

Bedin, L., King, I., Anderson, J., \& Piotto, G. 2001, ApJ, 560, L75

Bennett, C., Halpern, M., Hinshaw, G., et al. 2003, ApJS, 148, 1

Bergeron, P., Leggett, S., \& Ruiz, M. 2001, ApJS, 133, 409

Bergeron, P., Saumon, D., \& Wesemael, F. 1995, ApJ, 443, 764

Carney, B. 1996, PASP, 108, 900

Carretta, E., \& Gratton, R. 1997, A\&AS, 121, 95

Carretta, E., Gratton, R., Clementini, G., \& Fusi Pecci, F. 2000, ApJ, 533, 215

Cassisi, S., Castellani, V., Ciarcelluti, P., Piotto, G., \& Zoccali, M. 2000, MNRAS, 315, 679

Cayrel, R., Hill, V., Beers, T. C., et al. 2001, Nature, 409, 691

Chabrier, G. 1999, ApJ, 513, L013

Chabrier, G. 2001, ApJ, 554, 1274

Chabrier, G., Brassard, P., Fontaine, G., \& Saumon, D. 2000, ApJ, 543, 216

D’Antona, F. 1998, in The Stellar Initial Mass Function, ed. G. Gilmore, \& D. Howell (San Francisco: ASP), ASP Conf. Ser., 142,157

Delfosse, X., Forveille, T., Ségransan, D., et al. 2000, A\&A, 364, 217

De Marchi, G., Nota, A., Leitherer, C., Ragazzoni, R., \& Barbieri, C. 1993, ApJ, 419, 658

De Marchi, G., Paresce, F., \& Portegies Zwart, S. 2004, A\&A, in preparation

Djorgovski, S. 1993, in Structure and Dynamics of Globular Clusters, ed. S. Djorgovski, \& G. Meylan (San Francisco: ASP), ASP Conf. Ser., 50, 373

Dominguez, I., Chieffi, A., Limongi, M., \& Straniero, O. 1999, ApJ, 524,226

Fall, M., \& Rees, M. 1985, ApJ, 298, 18

Fontaine, G., Brassard, P., \& Bergeron, P. 2001, PASP, 113, 409

Gratton, R., Bragaglia, A., Carretta, E., et al. 2003, A\&A, 408, 529

Gratton, R., Fusi Pecci, F., Carretta, E., et al. 1997, ApJ, 491, 749

Gnedin, O. Y., Lahav, O., \& Rees, M. J. 2001 [astro-ph/0108034]

Hansen, B. 1998, Nature, 394, 860

Hansen, B. 1999, ApJ, 520, 680

Hansen, B., Brewer, J., Fahlman, G. G., et al. 2002, ApJ, 574, L155

Harris, W. 1996, AJ, 112, 1487

Hook, R., \& Fruchter, A. 2000, in Astronomical Data Analysis Software and Systems IX, ed. N. Manset, C. Veillet, \& D. Crabtree (San Francisco: ASP), ASP Conf. Ser., 216, 521 
Ibata, R. A., Richer, H. B., Fahlman, G. G., et al. 1999, ApJS, 120, 265

Jorgensen, U., Hammer, D., Borysow, A., \& Falkesgraad, J. 2000, A\&A, 361, 283

Leggett, S., Ruiz, M., \& Bergeron, P. 1998, ApJ, 497, 294

Kanatas, I., Griffiths, W., Dickens, R., \& Penny, A. 1995, MNRAS, 272, 265

King, I., Anderson, J., Cool, A., \& Piotto, G. 1998, ApJ, 492, L37

Krauss, L. M. 2001, in International Conference on the Identification of Dark Matter, ed. N. Spooner, \& V. Kudryavtsev (Singapore: World Scientific), 1

Lahav, O. 2001, in Astrophysical Ages and Timescale, ed. T. von Hippel, C. Simpson, \& N. Manset (San Francisco: ASP), ASP Conf. Ser., 245, 617

Mestel, L. 1952, MNRAS, 112, 583

Micol, A., \& Durand, D. 2002, ST-ECF Newsletter, 30, 14

Mobasher, B. 2002, HST Data Handbook, ed. B. Mobasher (Baltimore: STScI)
Montalban, J., D’Antona, F., \& Mazzitelli, I. 2000, A\&A, 360, 935

Mould, J. 1998, Nature, 395, A20

Paresce, F., \& De Marchi, G. 2000a, ApJ, 543, 870

Paresce, F., \& De Marchi, G. 2000b, in Star formation from the small to the large scale, ESLAB Symp. 33 ESASP 445, ed. F. Favata, A. Kaas, \& A. Wilson (Noordwijk: ESA), 279

Pont, F., Mayor, M., Turon, C., \& VandenBerg, D. 1998, A\&A, 329, 87

Prada Moroni, P., \& Straniero, O. 2002, ApJ, 581, 585

Richer, H., Fahlman, G., Rosvick, J., \& Ibata, R. 1998, ApJ, 504, L91

Richer, H. B., Hansen, B., Limongi, M., et al. 2000, ApJ, 529, 318

Richer, H. B., Brewer, J., Fahlman, G. G., et al. 2002, ApJ, 574, L151

Straniero, O., Chieffi, A., \& Limongi, M. 1997, ApJ, 490, 425

Saumon, D., \& Jacobson, S. B. 1999, ApJ, 511, 107

von Hippel, T., \& Gilmore, G. 2000, AJ, 120, 1384 